\title{
Effects of Periodontal Therapy on the Management of Cardiovascular Disease ${ }^{+}$
}

\author{
Dorina Lauritano ${ }^{1, *}$, Giulia Moreo ${ }^{1}$, Francesco Carinci ${ }^{2}$, Alberta Lucchese ${ }^{3}$, Dario di Stasio ${ }^{3}$, \\ Fedora della Vella ${ }^{4}$ and Massimo Petruzzi ${ }^{4}$ \\ 1 Department of Medicine and Surgery, University of Milano-Bicocca, Centre of neuroscience, 20126 Milan, \\ Italy; moreo.giulia@gmail.com \\ 2 Department of Morphology, Surgery and Experimental medicine. University of Ferrara, 44121 Ferrara, \\ Italy; crc@unife.it \\ 3 Multidisciplinary Department of Medical-Surgical and Dental Specialties, University of Campania-Luigi \\ Vanvitelli, 80138 Naples, Italy; alberta.lucchese@unicampania.it (A.L.); dario.distasio@unicampania.it (D.d.S.) \\ 4 Interdisciplinary Department of Medicine, University of Bari, 70121 Bari, Italy; \\ fdellavella@gmail.com (F.d.V.); massimo.petruzzi@uniba.it (M.P.) \\ * Correspondence: dorina.lauritano@unimib.it; Tel.: +39-3356790163 \\ + Presented at the XV National and III International Congress of the Italian Society of Oral Pathology and \\ Medicine (SIPMO), Bari, Italy, 17-19 October 2019.
}

Published: 10 December 2019

Cardiovascular disease (CVD) is a common cause of death, representing $29 \%$ of the mortality all over the word. Estimates for 2006 show that CVD is one of the world's main cause of death, with 17.1 million death per year. More than 70 million Americans have been diagnosed with various forms of CVD, including high blood pressure, coronary artery disease (acute myocardial infarction and angina pectoris), disorders of peripheral arteries etc. There is strong evidence that periodontal disease (PD) is associated with an increased risk of CVD. In addiction many patients with CVD are also affected by PD, which can be mild or severe [1,2]. The aim of this manuscript is to investigate the effects of periodontal therapy on the management of CVD. 34 randomised controlled trials and reviews were included in this mauscript to test the effects of different periodontal therapies for patients with CVD. In conclusion we may affirm that there is some lack of knowledge on relations between PD and CVD, however there is sufficient evidence to justify a periodontal treatment to prevent CVD, in fact PD is very prevalent in middle-aged population and can have a significant impact on the cardiovascular function $[3,4]$.

Conflicts of Interest: The authors declare no conflict of interest.

\section{References}

1. American Academy of Periodontology. International workshop for classification of periodontal diseases and conditions. Ann. Periodontol. 1999, 4, 7-112.

2. Blum, A.; Front, E.; Peleg, A. Periodontal care may improve systemic inflammation. Clin. Investig. Med. 2007, 30, E114-E117.

3. Socransky, S.S.; Haffajee, A.D.; Cugini, M.A.; Smith, C.; Kent, R.L., Jr. Microbial complexes in subgingival plaque. J. Clin. Periodontol. 1998, 25, 134-144.

4. Luis, A.J. Atherosclerosis. Nature 2000, 407, 233-241.

(C) 2019 by the authors. Licensee MDPI, Basel, Switzerland. This article is an open access article distributed under the terms and conditions of the Creative Commons Attribution (CC BY) license (http://creativecommons.org/licenses/by/4.0/). 